Baltic Astronomy, vol. 9, 618-624, 2000

\title{
MASCOT: A MULTI-WAVELENGTH ALL-SKY CATALOGUE FOR OBSERVATIONS WITH GROUND- AND SPACE-BASED TELESCOPES
}

\author{
O. Dluzhnevskaya ${ }^{1}$, O. Malkov ${ }^{1}$ and F. Pasian ${ }^{2}$ \\ ${ }^{1}$ Institute of Astronomy, Pyatnitskaya Str. 48, Moscow 109017, Russia \\ ${ }^{2}$ Osservatorio Astronomico di Trieste, Via Tiepolo 11, Trieste 34131, Italy
}

Received September 22, 2000.

\begin{abstract}
New powerful observational techniques require a careful planning of observing programmes. These requirements will be met within the proposed project MASCOT, A Multi-wavelength All-Sky Catalogue for Observations with ground- and space-based Telescopes. MASCOT will be based on the world's largest digitized all-sky survey containing astrometric and photometric data, USNO-A V2.0, which will be cross-identified with catalogued astronomical objects (extragalactic sources, variable stars, double stars, pulsars, high proper motion stars, nearby stars, Solar System objects, Hipparcos/Tycho catalogue objects) as well as with major non-optical (radio, IR, UV, $\mathrm{X}$-ray) surveys. A world-wide access to MASCOT will be provided via standard Internet tools.
\end{abstract}

Key words: methods: data analysis - catalogs - surveys

\section{INTRODUCTION}

Astronomers need to know the exact coordinates of objects under study and other data possibly already existing on these objects in order to carry out proper identification and quick interpretation of objects' properties. Predictions of number density of objects with given properties are highly desirable for planning new observational facilities and space missions. The proposed project, a Multi-wavelength 
All-Sky Catalogue for Observations with ground- and space-based Telescopes (hereafter MASCOT)

- will be based on the USNO-A V2.0 (hereafter USNO-A2), which will be cross-identified with catalogued data on important astronomical objects;

- will be world-wide accessible via standard Internet tools.

The main goals of the project are

- to classify the USNO-A2 objects and to construct a multiwavelength all-sky catalogue for preparing and carrying out observations with ground- and space-based telescopes, for quick identification and interpretation of observations;

- to supply the data centers and other holders of basic astronomical data sets with accurate positions, multicolor photometry, pulsar timing, VLBI parameters and other data extracted from the MASCOT for relevant objects.

The following procedure is suggested to carry out the project:

- development and application of methods for cross-identification between the USNO-A2 and other general astronomical catalogues and data sets;

- design of tools for visualization and a www-based mechanism for remote access to the MASCOT.

\section{BACKGROUND AND JUSTIFICATION FOR UNDERTAKING THE PROJECT}

For the planning of a comprehensive present-day experiments, as a rule, observers need the following components (they are partly available to the astronomical community as separate high-quality products):

- a list of reference objects, having accurate coordinates and brightnesses (two huge all-sky catalogues with accurate coordinates are published in machine-readable format, namely USNOA2 - about 500 mln. objects and Guide Star Catalog, GSC about $20 \mathrm{mln}$. objects);

- cross-identification with objects from relevant astronomical catalogues (cross-identification of about 1 million objects brighter than $9 \mathrm{mag}$ from more than 50 basic astronomical catalogues is stored in the SIMBAD database). 
However, for the planning of present-day ground-based and spacebased observations this information is, as a rule, insufficient. In order to meet nowadays requirements, one needs to integrate these data, to incorporate new information on variable, binary and high proper motion stars and extragalactic sources, and extend them beyond optical range to the whole spectrum.

The USNO-A2 catalogue

- contains astrometric (positions in the ICRS) and photometric (magnitudes in two bands) information for 526280881 objects;

- contains all sources found to be coincident between the blue and red survey plates;

- lacks object classification;

- is only identified with the GSC: 19524809 (brightest) USNO-A2 objects are flagged as GSC objects.

Cross-identifications (XID) with other astronomical catalogues and lists of non-stellar and the most interesting stellar objects as well as with sources from most important non-optical surveys are still missing. Consequently, little is known both about the nature of these objects themselves and about their relations to other data already catalogued and independently available in machine-readable form.

In order to use all this information in the effective way, a multiwavelength all-sky catalogue, and corresponding tools providing easy and intuitive remote access to the scope of the data are highly desirable. These are the main reasons for the construction of the MASCOT.

The immediate use of the MASCOT is the quick interpretation of observational data, and the possible detection of unexpected objects and phenomena. The MASCOT containing position, multicolor photometry and object classificators for about 500000000 objects down to $20-21 \mathrm{mag}$ will provide statistically reliable samples. The vast majority of objects in various optical and non-optical catalogs, databases, lists and surveys (hereafter basic astronomical data set, BADS) are, in principle, registered on POSS-I, SERC and ESO plates and included (though, not identified) in the USNO-A2. However, the positions currently listed in the BADS are often both heterogeneous and in many cases far inferior to the USNO-A2 precision. Merging the objects from the USNO-A2 with the BADS will improve both sets of data. 


\section{RESEARCH PROGRAMME}

1. The first step of the project is the cross-identification (XID) of USNO-A2 objects with the BADS for optical objects. BADS is expected to include data from available catalogues and lists of the following types of objects which will be flagged in the USNO-A2 (the main BADS and the approximate number of objects are given in parenthesis):

1.1. Extragalactic objects (NASA/IPAC Extragalactic Database, NED; 800000 ),

1.2. Variable stars (General Catalogue of Variable Stars, GCVS; $25000)$,

1.3. Double stars (Washington Visual Double Star Catalog, WDS; 80000 ),

1.4. Pulsars (Princeton Catalogue, EPN databases; 1000),

1.5. High proper motion stars (New Luyten catalogue of Stars with Proper Motions Larger than Two Tenths of an Arcsecond, NLTT; 60000),

1.6. Nearby stars (Catalogue of Nearby Stars, CNS3; 4000),

1.7. Solar System objects (New Minor Planet Database in USNO, MPD; 1000),

1.8. The stars with high precise and homogeneous coordinates, proper motions and two-color photometry (catalogues of Hipparcos and Tycho missions, 2500000).

BADS data will be cross-matched with information from on-line data services, such as the SIMBAD database. The identification process will be carried out in three different modes:

A1. The Identification of variable stars with usually badly known coordinates and photometry requires semi-manual processing by experts; the same approach will be applied to high proper motion stars and minor planets which change their positions with time.

B1. Extragalactic objects have, as a rule, a larger set of parameters available from NED, and could be identified automatically in a multi-dimensional parameter space using artificial intelligence techniques.

C1. XID of double stars, pulsars, stars from Hipparcos/Tycho catalogues and nearby stars with usually sufficiently accurate coor- 
dinates is unlikely to require the involvement of experts and will be made automatically by means of XID software.

2. The second step of the project is XID of USNO-A2 objects with the BADS for non-optical objects. Below we list particular ranges of the electromagnetic spectrum and the principal BADS for these ranges together with approximate number of objects.

2.1. Radio data (IERS and Green Bank radio surveys, 10000),

2.2. Infrared data (IRAS PSC, 600000 ),

2.3. Ultraviolet data (IUE archive, 100000 ; also Rosat [Extreme Ultraviolet Survey] and EUVE missions),

2.4. X-ray data (Rosat All-Sky Survey [RASS], 80000 ).

The XID of non-optical sources is generally more difficult due to their large positional errors. The identification process will be carried out in three different modes:

A2. Some of non-optical objects are already identified with optical counterparts (IRAS PSC, RASS). The XID of those counterparts will be carried out in accordance with modes set for optical sources.

B2. Identification rule sets will be constructed and applied to UV sources, similar to mode B1 (see above).

C2. The identification of reference radio sources from IERS and Green Bank sources list will be made under general rules in accordance with the precise positions of radio sources.

During the first two steps of the project the two additional goals will be achieved:

BADS of extragalactic sources (NED) and variables stars (GCVS) will be complemented by accurate positions and, if necessary, twocolor photometry from the USNO-A2. Concerning non-optical sources, the accurate positions for IR and X-ray sources from the USNO-A2 can be used by planning observations of space experiments within those spectral ranges as well as for ground-based IR projects.

The XID of minor planets whose positions are known with a very high accuracy gives us an exceptional opportunity for a detailed investigation of some statistical properties of the USNO-A2. We will be able to detect possible systematic biases in USNO-A2 positions in different regions of the sky.

3. The purpose of the third step is to build up a multiwavelength all-sky catalogue (MASCOT), including data from the USNO-A2, as well as several flags indicating the results of the XID 
and their reliability. It will be possible to query the MASCOT both by coordinates and object identifiers, with subsequent visualization of the sampled sky area.

Within this step, we propose to design tools of visualization of the MASCOT for the planning, preparation and carrying out of observations, quick identification of objects and interpretation of results.

Finally, an appropriate user interface allowing access to the MASCOT and supporting the access via Internet and www will be designed. A comprehensive and intuitive html- and Java-based interface for the MASCOT will be implemented. The user will be able to browse the MASCOT specifying constraints and consequently retrieve information on the objects. Graphic and image display with a certain amount of interactivity will be possible. The Astronomical Server URL (ASU) standard will be used to guarantee proper access to the catalogue from the Internet. The MASCOT will be set at Institute of Astronomy, Moscow (INASAN) and made available via Internet to the astronomical community.

\section{RESEARCH TEAMS AND TASKS}

The above tasks will be shared among the participating teams in accordance with the program defined in the following.

Osservatorio Astronomico di Trieste (OAT): project coordination, management and administration; design of the www interface to the final catalogue.

Centre de Données astronomiques de Strasbourg (CDS): scientific support during the XID phase; local support (database and tools) to localize different objects; managing of the information on the MASCOT in the Astroweb directory of Internet resources.

Institute of Astronomy, Moscow (INASAN): XID of the USNOA2 catalog with extragalactic objects, UV-sources and high proper motion stars; construction and management of the MASCOT; design and development of the www interface to the MASCOT.

Sternberg Astronomical Institute, Moscow (SAI): XID of the USNO-A2 catalog with variable stars and nearby stars.

Pulkovo Astronomical Observatory, St.-Petersburg: XID of the USNO-A2 catalog with minor planets and double stars; investigation of positional biases and random errors in the USNO-A2. 
Main Astronomical Observatory, Kiev (MAO): XID of the USNO-A2 catalog with the Hipparcos/Tycho stars, IR- and X-ray sources.

Puschino Radioastronomical Observatory (PRAO): XID of the USNO-A2 catalog with pulsars and radio sources.

\section{CONCLUSIONS}

The main goals of the proposed project are to classify the USNOA2 objects and to construct a multi-wavelength all-sky catalogue for preparing and carrying out observations with ground- and spacebased telescopes, for quick identification and interpretation of observations; and to supply the data centers and other holders of basic astronomical data sets with accurate positions, multicolor photometry and other data extracted from the MASCOT for relevant objects. The following products will be made available: a multi-wavelength all-sky catalogue for observations with ground- and space-based telescopes; XID of the USNO-A2 catalog with other astronomical catalogues; accurate positions for NED, GCVS and non-optical objects. 